\title{
MUSikWettbeWerb VS. WettbeWerbsmusik - DAS Dilemma des EuRovision SONG CONTESTS
}

\author{
Irving Wolther
}

Der Eurovision Song Contest (ESC) ist der weltweit größte internationale Wettbewerb für populäre Musik. Weit über die Grenzen Europas hinaus wird der Liederwettstreit Jahr für Jahr von bis zu 600 Millionen Menschen verfolgt. Seit seiner ersten Ausrichtung in Lugano (Schweiz) im Jahr 1956 ist die Anzahl der teilnehmenden Nationen von sieben auf über 40 angewachsen. Das Interesse an einer Song-Contest-Teilnahme ist ungebrochen. Insbesondere Länder des ehemaligen Ostblocks bemühen sich um eine Aufnahme in die stetig wachsende ESC-Gemeinde.

Angesichts dieser Tatsachen wäre davon auszugehen, dass der ESC eine ideale Plattform für die Förderung und europaweite Vermarktung nationaler Musikproduktionen darstellt. Tatsächlich ist die Musikförderung als Zielsetzung fest im Wettbewerbsreglement verankert:

»The purpose of the Contest is to promote high-quality original songs in the field of popular music, by encouraging competition among artists, songwriters and composers through the international comparison of their songs" (European Broadcasting Union [EBU] 2002: 1).

Den hehren Absichten seiner Gründerväter zum Trotz wurde dem ESC in der Vergangenheit immer wieder vorgeworfen, den aktuellen musikalischen Entwicklungen hinterher zu hinken. Der enorme finanzielle Aufwand, der für diese Veranstaltung betrieben werde, sei angesichts ihrer kommerziellen Bedeutungslosigkeit nicht gerechtfertigt. So stellt der schwedische Musikwissenschaftler Alf Björnberg fest:

»[A]lthough the extensive exposure offered by the contest may help to bring even relative commercial failures up to the break-even point, the number of big international hits, of the magnitude necessary to keep transnational music industry rolling, to come out of the ESC throughout its history is surprisingly small« (Björnberg 1989: 377). 
Aus der relativen kommerziellen Erfolglosigkeit der Wettbewerbsbeiträge zogen Kritiker das Fazit, dass die beim ESC vorgestellte Musik altmodisch und deshalb nicht konkurrenzfähig sei. Insbesondere in der Bundesrepublik Deutschland, die traditionell über eine stark exportorientierte Musikindustrie verfügt, hatten die Plattenfirmen den ESC »längst abgeschrieben« (Meier-Beer 2002: 418). So zielten die Bemühungen um eine Modernisierung der Veranstaltung, die vor allem auf bundesdeutsche Initiative vorangetrieben wurden, vordergründig darauf, den Wettbewerb für die Musikwirtschaft attraktiver zu machen und auf diese Weise für eine größere Zuschauerresonanz zu sorgen.

Es ist verwunderlich, dass ein Musikwettbewerb, der sich erklärtermaßen der Musikförderung verschrieben hat, von den deutschen Plattenfirmen »abgeschrieben« worden war. Wie ist diese Entwicklung zu erklären?

\section{Der Eurovision Song Contest in Deutschland}

Weder in der Presse noch seitens der Musikindustrie wurde in Deutschland jemals mit Kritik am ESC gespart. Dabei stand vor allem die Frage nach dem musikalischen und textlichen Anspruch der eigenen Wettbewerbsbeiträge im Mittelpunkt der Diskussion. Bereits 1963 urteilte die Branchenzeitschrift Der Musikmarkt über die deutsche Vorentscheidung: „Alle zur Wahl stehenden Stücke waren typische Weder-noch-Lieder. Einerseits wollte man etwas mit Niveau schreiben. Andererseits sollte es aber keinesfalls zu hoch sein« (Götze 1963). Ein Jahr später wurde bereits von dem für die Bundesrepublik »gewohnten Fiasko « gesprochen (zit. n. Musikmarkt 1989: 36), Kritiker bezeichneten den ESC im Zusammenhang mit der ursprünglich geläufigen Bezeichnung Grand Prix Eurovision gar als »Grand malheur« (Linke 1972: 255). In der Tat ist die Erfolgsbilanz bundesdeutscher Vertreter beim ESC eher bescheiden. 1982 gelang Sängerin Nicole mit dem schon fast legendären »Ein bißchen Frieden« der erste und einzige ESC-Sieg für Deutschland, insgesamt fünfmal (1964, 1965, 1974, 1995 und 2005) landeten die bundesdeutschen Vertreter auf dem letzten Platz. Ein enttäuschendes Bild, wenn man sich die Bedeutung des deutschen Musikmarktes in der Welt vor Augen hält. Doch warum tut sich Deutschland so schwer mit dem ESC? Um dieser Frage auf den Grund zu gehen, ist es erforderlich, einen Blick auf seine Entstehungsgeschichte zu werfen. 


\section{Die Entstehungsgeschichte des Eurovision Song Contests}

Ursprünglich war der Wettbewerb ins Leben gerufen worden, um einen jährlich wiederkehrenden Anlass für die Zusammenarbeit der europäischen Fernsehanstalten zu schaffen. Zu diesem Zweck wurde nach dem Vorbild des bereits seit 1951 existierenden Festival della Canzone Italiana (Festival di Sanremo) ein Komponistenwettbewerb ins Leben gerufen. Dieser wurde 1956 erstmalig ausgestrahlt.

Obwohl die Musikförderung eines der zentralen Anliegen des Wettbewerbs war, blieben die Belange der Musikindustrie bei der Gestaltung des Reglements weitgehend unberücksichtigt. Zwar wurde den Musikverlegern großzügig gestattet, den Vermerk »Grand Prix Eurovision de la Chanson« auf der Plattenhülle des Siegertitels unterzubringen (was in späteren Jahren auch auf die übrigen veröffentlichten Beiträge ausgedehnt wurde), doch der ESC blieb in erster Linie eine Fernsehsendung, aus der die Musikindustrie erst im Anschluss ihren Nutzen ziehen durfte. Die mit der Organisation einer solchen Fernsehsendung verbundenen Zwänge wirkten sich zudem auf den Musikstil aus, der für die Veranstaltung charakteristisch werden sollte.

\section{Drei Minuten Länge}

Zunächst einmal wurde die Dauer der Wettbewerbsbeiträge auf drei Minuten beschränkt, um den Zeitrahmen der Veranstaltung bei stetig wachsender Teilnehmerzahl nicht zu sprengen. ${ }^{1}$ Je mehr Länder an der Veranstaltung teilnahmen, desto strikter wurde über die Einhaltung dieser Regel gewacht; bei Missachtung drohte die Disqualifikation. Das Drei-Minuten-Format erwies sich für Kompositionen nach dem A-B-A-B-C-B-Schema (Strophe-RefrainStrophe-Refrain-Bridge-Refrain) als besonders geeignet. Für musikalische Formen, die auf umfangreiche Intros und Instrumentalsoli Wert legten (u.a. Rockmusik), kam diese zeitliche Begrenzung jedoch einem Ausschlusskriterium gleich.

1 Im ursprünglichen Reglement waren zwar drei bis dreieinhalb Minuten vorgesehen, doch einzelne Beiträge erreichten live eine Länge von bis zu sieben Minuten (»Chorde della mia chitarra« von Nunzio Gallo für Italien 1957). 


\section{Sechs Personen auf der Bühne}

Die Anzahl der auf der Bühne anwesenden Musiker und Tänzer wurde zunächst auf zwei, später auf sechs Personen begrenzt, um die zwischen den Beiträgen erforderlichen Umbaupausen möglichst kurz zu halten. Ab 1971 wurde der Wettbewerb auch für Musikgruppen geöffnet. ${ }^{2}$ Da die seit 1964 in Europa verbreitete Beatmusik vorwiegend durch Gruppen repräsentiert wurde, brachte diese Regeländerung (mit einigen Jahren Verspätung) eine neue Musikfarbe in den ESC. Zwar blieb die klassische Rockmusik weiterhin außen vor, doch waren in den 1970er Jahren zahlreiche international erfolgreiche Popgruppen im Starterfeld des Wettbewerbs zu finden (ABBA, Baccara, Silver Convention, The Shadows).

\section{Live-Orchester}

Einen weiteren reglementspezifischen Faktor stellte der Einsatz eines LiveOrchesters dar. Anstelle der ursprünglich geplanten individuellen Inszenierung der einzelnen Beiträge durch verschiedene Regisseure war den Ländern bzw. den teilnehmenden Fernsehanstalten die Orchesterleitung durch eigene Dirigenten gestattet worden. Auch als die Technik des Playback bereits in die meisten anderen europäischen Unterhaltungsprogramme Einzug gehalten hatte, blieben Einspielungen vom Band nur insoweit zulässig, als die Instrumente auch auf der Bühne sgespielt < wurden. Dies war beispielsweise bei Beiträgen erforderlich, die seltene, landestypische Instrumente verwendeten, die von den Angehörigen der Rundfunkorchester nicht beherrscht wurden.

Die Auswirkungen der Orchesterpflicht auf die musikalische Entwicklung der Beiträge waren vielfältig. Ein sanftes Ausklingen der Titel (Fade Out), das bei den meisten Pop-Produktionen üblich war, konnte das Orchester nicht leisten; alle Beiträge mussten mit einem Cold End versehen werden. Zudem wurden Beiträge mit hymnischem Charakter favorisiert, da sie die instrumentale Klangfülle des Live-Orchesters zu ihrem Vorteil einsetzen konnten. Moderne Pop- und Rockarrangements waren dagegen zunehmend schwieriger umzusetzen, da viele spezifische Klangeffekte durch das

2 Manche Länder behalfen sich vor der entsprechenden Regeländerung mit dem Auftritt von Gesangsduos, die von den übrigen Gruppenmitgliedern als Choristen begleitet wurden, so zum Beispiel das jugoslawische Quintett Dubrovački Trubaduri, das 1968 unter dem Namen Luči Kapurso und Hamo Hajdarhodžić mit dreiköpfigem Chor auftrat. 
Orchester nicht wiedergegeben werden konnten. So unterschieden sich die Live-Versionen der ESC-Beiträge in den 1980er und 1990er Jahren meist deutlich von den Studioversionen, allerdings nicht immer zum Nachteil der Komposition. Viele Autoren und Arrangeure waren froh, für die Darbietung ihrer Titel die umfangreichen Möglichkeiten eines Orchesters nutzen zu können, das ihnen in ihrer Heimat aus Kostengründen nie zur Verfügung gestanden hätte. 1998 wurde schließlich die Verwendung von Halbplayback zugelassen, nicht zuletzt um die Kosten der Veranstaltung zu reduzieren, denn den Luxus eines eigenen Rundfunkorchesters leisteten sich zu diesem Zeitpunkt schon längst nicht mehr alle Fernsehanstalten. Der Unterschied zwischen Live-Performance und verkaufsfertigem musikalischen Produkt wurde so auf ein Minimum reduziert - auch zur Freude der Plattenfirmen, für die das Orchester einen schwer kalkulierbaren Risikofaktor darstellte.

\section{Vortrag in Landessprache}

Der Zwang zum Vortrag des Beitrags in Landessprache trug maßgeblich zu der Entwicklung einer charakteristischen Musikfarbe innerhalb des ESC bei. Auf Grund der unterschiedlichen Entwicklung der nationalen Musikmärkte, war ein breitgefächertes Musikangebot in Nationalsprache in vielen Ländern nicht mehr selbstverständlich. So kam es, dass vorzugsweise Beiträge eines bestimmten Genres eingesandt wurden, andere Musikfarben hingegen nur selten oder überhaupt nicht vertreten waren. Die Bemühung um musikalische Ausdrucksformen, die in ganz Europa erfolgreich sein sollten, führte zudem in einigen Ländern zur Entstehung eines spezifischen Musikstils, der von geringer Originalität und Experimentierfreude gekennzeichnet ist. Björnberg (1989: 376) weist darauf hin, dass in seiner Heimat eine bestimmte Form »kontinentaler Popmusik« als prototypisch für einen guten schwedischen ESC-Beitrag empfunden wird. Dennoch gab es immer wieder Beispiele für den Willen, die eigene Musikkultur in typischer Weise zu repräsentieren, vor allem aus den Ländern des Mittelmeerraums und Osteuropas.

Der Wegfall der Sprachenregelung 1999 hatte zunächst zur Folge, dass der Großteil der teilnehmenden Kompositionen auf Englisch vorgetragen wurde. Hatten sich Länder mit vermeintlich unmelodischer Landessprache zuvor bemüht, ihre Beiträge durch Internationalismen seuropakompatibel zu gestalten, ${ }^{3}$ sahen sie nun in der englischen Sprache ein Mittel, um den von ihnen lang ersehnten Erfolg zu erzielen. Dabei wurde der Titel bei der

3 Erfolgreichste Beispiele sind die Siegertitel »La, la, la« der Sängerin Massiel für Spanien (1968), »Diggy-loo, Diggi-ley« der Gruppe Herrey's für Schweden (1984) und »Diva« der Interpretin Dana International für Israel (1998). 
nationalen Vorauswahl häufig in der landessprachlichen Version gesungen, um dann im internationalen Finale mit englischem Text vorgetragen zu werden. Der rumänische Sänger Dan Teodorescu begründet die Wahl der englischen Sprache für seinen Titel »Luna « wie folgt: »Wir mussten einen Kompromiss eingehen. [...] Rumänisch klingt für die meisten Menschen fremd. Sobald sie aber englische Worte verstehen, denken Sie: >Ach, die Rumänen sind doch zivilisierte Menschen! « (Wolther 2000).

Bezeichnend ist in diesem Zusammenhang, dass häufig Künstler am ESC teilnehmen, die in ihrer Heimat in Landessprache singen und nur ihren ESCAuftritt in englischer Sprache absolvieren, um sich auf diese Weise neue Absatzmärkte zu erschließen. Die zum Teil haarsträubend schlechte Aussprache des Englischen wird allgemein als einer der Gründe angeführt, weshalb der ESC als Popmusikwettbewerb nicht ernst genommen wird.

\section{Nationalspezifische Musikvorlieben}

Musikalisch sollte die Möglichkeit eines Vortrags in englischer Sprache zu einer >Verjüngung < der dargebotenen Beiträge führen. Doch die Vorstellungen von zeitgemäßer Musik variieren von Musikmarkt zu Musikmarkt beträchtlich. ${ }^{4}$ Es war in der Vergangenheit durchaus nicht der Fall, dass die Spitzenpositionen in den europäischen Verkaufshitparaden vorrangig von Rock- oder Pop-Interpreten aus den USA und England belegt wurden. Die musikalischen Präferenzen in den einzelnen Ländern besaßen jenseits der angloamerikanischen Produktpalette sehr unterschiedliche Charakteristika, auch hinsichtlich der dominierenden Musikfarbe. So können bestimmte Darbietungen für das Publikum in einem Land sehr >modern in den übrigen Ländern aber möglicherweise altmodisch oder sogar peinlich gewirkt haben. Björnberg (1989: 377) weist darauf hin, dass die Presse in Schweden mit Unverständnis, zum Teil sogar mit heftigem Unmut auf ESCBeiträge reagierte, die den stilistischen Erwartungen der Journalisten nicht entsprachen. Eine Bewertung der musikalischen Entwicklung des Wettbewerbs aus der Sicht einer bestimmten Nation ist somit problematisch.

4 Ein sehr anschauliches Beispiel für den Erfolg >unzeitgemäßer Musik bietet "Schnappi, das kleine Krokodil«, das die deutschen Verkaufshitparaden 2004/ 2005 über viele Wochen anführte. 


\section{Eurovision Song Contest $=$ Schlager?}

Dennoch ist nicht von der Hand zu weisen, dass durch die vorgenannten reglementbedingten Einschränkungen im Rahmen des ESC nur ein bestimmter Ausschnitt der musikalischen Entwicklung in Europa vorgestellt werden konnte. Die spezifische Form von Unterhaltungsmusik, die den Wettbewerb dominierte, wurde aus bundesdeutscher Sicht zunächst als Chanson, später als Schlager empfunden. War dieser Begriff in den 1960er und frühen 1970er Jahren noch vornehmlich positiv besetzt, so wandelte er sich in den 1980er und 1990er Jahren zu einem Synonym für qualitativ minderwertige Musik mit primitiven Arrangements und einfallslosen Texten. Die Vorschriften des ESC-Reglements wurden somit aus deutscher Sicht zu einem Problem, da sie die Entstehung musikalischer Produktionen begünstigten, für die es in der Bundesrepublik außer dem Schlager kein Äquivalent gab. Der Schlager indes erfüllte nicht mehr den qualitativen Anspruch an nationalsprachige Unterhaltungsmusik, der in anderen Ländern selbstverständlich war. So wandelte sich der ESC im deutschen Sprachgebrauch zum »Schlager-Grand-Prix « - mit allen negativen Konnotationen, die der Begriff beinhaltet. Entsprechend schwierig gestaltete sich die Vermarktung von ESC-Beiträgen, die sich dem Schlager nicht eindeutig zuordnen ließen. Schließlich kamen in Deutschland selbst die Siegertitel erst mit monatelanger Verspätung auf den Markt, weil sich keine Plattenfirma für eine Veröffentlichung bereit fand.

\section{»Anspruch«}

Ein weiteres Problem lag in dem Qualitätsanspruch, der seitens der Fernsehverantwortlichen an den bundesdeutschen ESC-Beitrag gestellt wurde. So erklärte der Intendant des Hessischen Rundfunks, Hans-Otto Grünefeldt, den Fernsehzuschauern bei der deutschen ESC-Vorentscheidung 1970: »Es geht nicht darum [...] ein Lied herauszufinden, das etwa kommerziell die größten Chancen hätte [...]. Es geht darum, das Lied auszuwählen, das auf [...] diesem speziellen Grand Prix Eurovision für die ARD bestehen kann« (Hessischer Rundfunk 1970). Für den Fernsehmacher stand kommerzieller Erfolg im Widerspruch zu den erhofften Siegchancen. Wichtiger als einen Hit zu landen, war in jedem Fall, sich international nicht zu blamieren.

Doch auch Komponisten und Texter zeigen sich im Zusammenhang mit dem ESC befangen von abstrakten Qualitätsvorstellungen, wie der Texter 
Bernd Meinunger im Interview gesteht. Einfache Liebeslieder seien zu banal für einen deutschen Beitrag, darum müsse es um »Weltbewegendes« gehen:

»Das Damoklesschwert des Anspruchs schwebt immer über dem Komponisten, dem Texter, dem Produzenten. [...] Man hat in diesem Fall auch eine Art nationale Verantwortung: Man will sein Land ja nicht mit einem 08/15-Lied über eine Liebesbeziehung abstinken lassen. «5

Offensichtlich gibt es auch beim Publikum eine konkrete Vorstellung davon, wie ein angemessener ESC-Titel zu klingen hat und von wem er dargeboten werden soll. In der Bundesrepublik Deutschland schien 2001 mit der Ankündigung der Teilnahme von Big-Brother-Star Zlatko der Sieger bereits festzustehen, doch das Publikum buhte den vormals populären Interpreten aus, weil sein dilettantischer Vortrag offenbar nicht der Vorstellung einer adäquaten Repräsentation Deutschlands beim ESC entsprach - Zlatko landete nur auf Platz 6. Die Auswahl eines ESC-Beitrags unterliegt also anscheinend einer Reihe von Kriterien, die mit den ökonomischen Interessen der Musikindustrie nicht zwangsläufig vereinbar sind. Selbst die Presse kann sich von bestimmten Qualitätsvorstellungen nicht freimachen, wie Helmut Scherer und Daniela Schlütz in ihrer Studie zur ESC-Vorentscheidung 2000 feststellen: »Für einige Journalisten ist der Grand-Prix-Vorentscheid ein Ereignis von nationaler Bedeutung, da der Gewinner als Botschafter Deutschlands im internationalen Wettbewerb auftritt« (Scherer/Schlütz 2003: 54).

\section{Auswirkungen}

Der hohe Erwartungsdruck, der gegenüber dem nationalen ESC-Beitrag existiert, mag häufig (und nicht nur in Deutschland) ein Grund für die Zurückhaltung der Plattenfirmen gewesen sein, bekannte Künstler in den Wettbewerb zu entsenden. Möglicherweise werden aus diesem Grund mittlerweile verstärkt die Gewinner von Casting-Shows ins Rennen um den Grand Prix geschickt: Junge Talente haben keinen Ruf zu verlieren und können von einem guten Abschneiden nur profitieren. So konnte Casting-Show-Sieger Max Mutzke mit seiner ESC-Teilnahme 2004 den Grundstein für seine Karriere legen, und auch 2005 ging mit Gracia Baur eine ehemalige CastingShow-Teilnehmerin für die Bundesrepublik Deutschland an den Start. ${ }^{6}$

5 Bernd Meinunger in einem Interview mit dem Verfasser, geführt am 27.4.1994 - drei Tage vor dem ESC 1994 in Dublin.

6 Weitere Beispiele für diese Entwicklung finden sich unter anderem in Frankreich, Irland, Portugal und Spanien. Allerdings ist das spanische Fernsehen mittlerweile davon abgerückt, den frisch gekürten Sieger der Casting-Show Opera- 
Auch wenn in der Vergangenheit ein schlechtes Abschneiden des Interpreten beim ESC nicht zwangsläufig einen Karriereknick nach sich zog, erwiesen sich schlecht platzierte Musiktitel doch zumeist als kommerzieller Flop. Eine Veröffentlichung des entsprechenden Tonträgers vor dem Wettbewerb war laut Reglement unzulässig. ${ }^{7}$ Komponisten und Textdichter vermieden es deshalb, kommerziell Erfolg versprechende Titel in den Wettbewerb zu entsenden, oder wie ein nicht genannter Komponist präzisiert: »Kein prominenter Autor dürfte [...] bereit sein, eine Spitzen-Nummer nur für eine einmalige Fernsehsendung zu opfern« (Linke 1972: 257). Mittlerweile dürfen die Beiträge schon bis zu einem halben Jahr vor dem ESC veröffentlicht werden und die Darbietung unterscheidet sich nur noch unwesentlich von der Studioproduktion. Insofern kann von einer Kommerzialisierungstendenz des Wettbewerbs gesprochen werden. Die Musikindustrie kann also zufrieden sein. Oder vielleicht doch nicht?

Bei den nationalen Vorentscheidungen werden noch immer nicht zwangsläufig die kommerziell erfolgreichsten Interpreten ins Rennen geschickt geschweige denn zum Sieger gekürt. Und selbst der Hauptinitiator der Reglementreform, NDR-Unterhaltungschef Jürgen Meier-Beer, bekennt sich klar dazu, dass er die Sendung nicht als Mittel zur Unterstützung der heimischen Musikindustrie versteht, sondern die Strukturen des Musikmarkts für eine bessere Vermarktung des Produkts Fernsehshow nutzen möchte: »Die Fernsehdramaturgie der nationalen Grand-Prix-Vorentscheidung ist direkt aus dem nationalen Musikmarkt zu ziehen [...]. Das Ziel der Musikförderung [...] bietet dem Fernsehmacher [...] keine Orientierungshilfe « (Meier-Beer 2002: 423). Auch nach 50 Jahren ist und bleibt der ESC also in erster Linie eine Fernsehsendung, deren Ziel vorrangig in der Erzielung hoher Einschaltquoten besteht. Die Förderung der nationalen Musikindustrien wird trotz aller Zugeständnisse der Programmgestalter immer hinter diesem Ziel zurückstehen.

ción Triunfo beim ESC zu sverheizen Interpreten zu schicken. Die Angst vor einer schlechten Platzierung, die der kaum begonnenen Karriere ein abruptes Ende setzen kann, scheint bei den verantwortlichen Produzenten relativ groß zu sein.

7 Dies führte zur Disqualifikation zahlreicher Wettbewerbstitel, u.a. des bundesdeutschen Beitrags »Hör den Kindern einfach zu« der blinden Sängerin Corinna May 1999. 


\section{Fazit}

Der Eurovision Song Contest ist ein von den Fernsehanstalten inszeniertes Medienereignis, das erst nachrangig musikökonomischen Zwecken dient. Die Anzahl der kommerziell erfolgreichen Kompositionen, die im Laufe der Jahre aus dem ESC hervorging, ist für eine musikalische Veranstaltung mit derartigem Stellenwert überraschend gering. Offenbar wurde durch das Reglement eine vorwiegend kommerzielle Orientierung des Wettbewerbs verhindert. Dies lässt sich auch daran erkennen, dass noch heute zahlreiche Wettbewerbsbeiträge nicht in den öffentlichen Verkauf gelangen.

Der enge Rahmen, der durch die Vorschriften des Reglements gesteckt wird, bestimmt, welcher Ausschnitt aus dem aktuellen Musikgeschehen innerhalb dieser Veranstaltung vorgestellt wird. Dieser Ausschnitt ist unter Umständen für die nationale Musikindustrie einzelner Länder kommerziell unattraktiv und führt dort zu einem nachlassenden Interesse der Musikindustrie und ihrer publikumswirksamen Interpreten am ESC. Da das Fernsehen aus der Popularität der teilnehmenden Beiträge unmittelbaren Nutzen in Form von Einschaltquoten zieht, wurde das Reglement dahin geändert, die Möglichkeiten zur Vermarktung der Wettbewerbsbeiträge zu verbessern. Das musikalische Grundgerüst der Veranstaltung blieb davon jedoch weitgehend unberührt, da es auf historischen Entwicklungen und Erwartungshaltungen beruht, die bei Fernsehverantwortlichen, Musikschaffenden und Publikum gleichermaßen verankert sind und sich den ökonomischen Interessen der Musikindustrie entziehen. Eine stärkere kommerzielle Orientierung des ESC im Sinne der Musikindustrie wird daher nur so weit möglich sein, wie sie die historisch gewachsene Erwartungshaltung an den Wettbewerb berücksichtigt.

\section{Literatur}

Björnberg, Alf (1989). »Music spectacle as ritual: The Eurovision Song Contest. «In: Proceedings of the IASPM 1989. Hg. v. Maison des Sciences de l'Homme. Paris: Maison des Sciences de l'Homme, S. 375-382.

European Broadcasting Union (EBU) (2002). Rules of the 2003 Eurovision Song Contest, 10. August, http://www.ebu.ch/departments/television/pdf/ESC2003 Rules_E.pdf?display=EN (Zugriff: 21.3.2005).

Götze, Werner (1963). "Schlagerbarometer 1963: Stark fallend.«In: Der Musikmarkt 52, 15.12.1963.

Hessischer Rundfunk (1970). Ein Lied für Amsterdam. Deutsche Vorentscheidung zum Grand Prix Eurovision de la Chanson 1970. Regie: Ekkehard Böhmer. Sendung vom 16. Februar. 
Linke, Norbert (1972). "Schlagerfestivals und -wettbewerbe." In: Schlager in Deutschland. Hg. v. Siegmund Helms. Wiesbaden: Breitkopf \& Härtel, S. 255262.

Meier-Beer, Jürgen (2002). »Making a Pop-Event.«In: Ein Lied kann eine Brücke sein. Die deutsche und internationale Geschichte des Grand Prix Eurovision. Hg. v. Jan Feddersen. Hamburg: Hoffmann \& Campe, S. 417-424.

Musikmarkt, Der (Hg.) (1989). 30 Jahre Single Hitparade. Starnberg \& München: Josef Keller Verlag.

Scherer, Helmut/Schlütz, Daniela (2003). Das inszenierte Medienereignis. Die verschiedenen Wirklichkeiten der Vorausscheidung zum Eurovision Song Contest in Hannover 2001. Köln: Herbert von Halem Verlag.

Wolther, Irving. (2000). »Rumänien.« In: Top of Music in Europe 33, S. 25.

\begin{abstract}
The Eurovision Song Contest (ESC) is the largest international contest for popular music in the world. Originally intended to strengthen the cooperation between European TV-stations, the aim to support the national music industries always played a secondary role. Apparently, its rules prevented the contest to develop in a more commercial direction.

The framework given by the rules of the ESC determines a particular selection of popular music presented at the show. This selection might be commercially less interesting than others in some of the participating countries, leading to a decreasing interest of the respective music industries and their most popular artists in the ESC. Since television takes advantage of the popularity of the participating entries due to increased viewing numbers, the rules have been changed accordingly in order to make commercial exploitation of the songs easier. The type of music presented at the show was nonetheless not much affected by these changes since it is based on historical developments and expectations firmly established in the minds of television executives, songwriters and viewers, defying the commercial interests of the music industry.
\end{abstract}

